https://helda.helsinki.fi

Co-evolution as an important component explaining microbial predator-prey interaction

\title{
Kaitala, Veijo
}

2020

Kaitala , V , Hiltunen , T , Becks , L \& Scheuerl , T 2020 , ' Co-evolution as an important component explaining microbial predator-prey interaction ' , Journal of Theoretical Biology , vol. 486,110095 . https://doi.org/10.1016/j.jtbi.2019.110095

http://hdl.handle.net/10138/321941

https://doi.org/10.1016/j.jtbi.2019.110095

acceptedVersion

Downloaded from Helda, University of Helsinki institutional repository.

This is an electronic reprint of the original article.

This reprint may differ from the original in pagination and typographic detail.

Please cite the original version. 


\title{
Co-evolution as an important component explaining microbial predator-prey interaction
}

\author{
Veijo Kaitala ${ }^{\mathrm{a}, 1, *}$, Teppo Hiltunen ${ }^{\mathrm{b}, \mathrm{c}}$, Lutz Becks ${ }^{\mathrm{d}}$, Thomas Scheuerl ${ }^{\mathrm{e}, 1}$ \\ a Organismal and Evolutionary Biology Research Programme, University of Helsinki, Helsinki, Finland \\ ${ }^{\mathrm{b}}$ Department of Food and Environmental Sciences / Microbiology and Biotechnology, University of Helsinki, P.O. Box 56, 00014, Finland \\ ${ }^{\mathrm{c}}$ Department of Biology, University of Turku, Turku, Finland \\ ${ }^{\mathrm{d}}$ Limnology - Aquatic Ecology and Evolution, Limnological Institute, University of Konstanz, Konstanz, Germany \\ e Amerang Str. 8, 83435 Bad Reichenhall, Germany
}

\section{A R T I C L E I N F O}

\section{Article history:}

Received 4 July 2019

Revised 1 November 2019

Accepted 26 November 2019

Available online 26 November 2019

\section{Keywords:}

Quantitative trait evolution model

Population dynamics

Microcosm

Pseudomonas

Tetrahymena

\begin{abstract}
A B S T R A C T
Predator-prey relationships belong to the most important and well-studied ecological interactions in nature. Understanding the underlying mechanisms is important to predict community dynamics and to estimate coexistence probability. Historically, evolution has been considered to be too slow to affect such ecological interactions. However, evolution can occur within ecological time scales, potentially affecting predator-prey communities. In an antagonistic pair-wise relationship the prey might evolve to minimize the effect caused by the predator (e.g. mortality), while the predator might evolve to maximize the effect (e.g. food intake). Evolution of one of the species or even co-evolution of both species in predator-prey relationships is often difficult to estimate from population dynamics without measuring of trait changes in predator and/or prey population. Particularly in microbial systems, where microorganisms evolve quickly, determining whether co-evolution occurs in predator-prey systems is challenging. We simulate observational data using quantitative trait evolution models and show that the interaction between bacteria and ciliates can be best explained as a co-evolutionary process, where both the prey and predator evolve. Evolution by prey alone explains the data less well, whereas the models with predator evolution alone or no evolution are both failing. We conclude that that ecology and evolution both interact in shaping community dynamics in microcosms. Ignoring the contribution of evolution might lead to incorrect conclusions.
\end{abstract}

(c) 2019 Published by Elsevier Ltd.

\section{Introduction}

Bacteria are an important prey source for various consumers such as bacteriophages and protozoan grazers and understanding such antagonistic interactions is critical to understand how microbial food webs work. Although microbial predator-prey interactions have been extensively studied (Abrams, 2000; Gause, 1934) and the ecological mechanisms ruling the dynamics are well understood (May, 1972; Rosenzweig, 1971; Volterra, 1926), we lack basic knowledge of how and when these interactions evolve (Abrams, 2000). Microbes are key organisms used in predator-prey experiments because they are easy to cultivate and time-series over multiple generations can be easily obtained under highly controlled conditions (Fussmann et al., 2007). Microbial organisms

\footnotetext{
* Corresponding author.

E-mail addresses: veijo.kaitala@helsinki.fi (V. Kaitala), teppo.hiltunen@helsinki.fi (T. Hiltunen), lutz.becks@uni-konstanz.de (L. Becks).

1 These authors contributed equally.
}

are famous for evolving quickly (Fiegna et al., 2015; Scheuerl and Stelzer, 2013; Wiser et al., 2013; Yoshida et al., 2003), which likely results in co-evolution (Buckling and Rainey, 2002; Scheuerl et al., 2019) and an evolutionary effect on any ecological interaction (Hairston et al., 2005; Schoener, 2011).

Because co-evolution is a highly complex process, and difficult to test in nature, our understanding of co-evolution is still limited (Friman and Buckling, 2013; Gómez and Buckling, 2011). Theoretical models are perfectly suited to test for co-evolutionary components in microbial predator-prey systems because traits otherwise difficult to estimate can be mathematically estimated (Mougi, 2010). Theoretical co-evolutionary models, including the quantitative genetics models (Abrams et al., 1993; Mougi, 2010; Mougi and Iwasa, 2011, and the references therein) have been studied intensively. However, no attempt, as far as we know; has been reported in analysing real data of apparently evolving microbial communities. In this case study we report such an attempt to model the evolutionary interaction of prey and predator in a con- 
trolled microcosm environment, which enables us to better understand when and how co-evolution affects antagonistic interactions.

A recent microcosm study (Hiltunen et al., 2018) investigated ancestral Pseudomonas fluorescens bacteria being predated by ancestral Tetrahymena thermophila ciliates. The communities were maintained by serial transfers for a period of 60 days and the population levels of the bacteria and ciliates were recorded. Using a quantitative genetics approach we analysed the observed predatorprey interactions of this study applying Lotka-Volterra model as a pure ecological version assuming no evolution (Volterra, 1926) and modified models (Mougi, 2010; Mougi and Iwasa, 2011) with one sided evolution or co-evolution.

Applying co-evolutionary modelling to real data allowed us to understand which evolutionary components determine such antagonistic interactions. Such models will be very valuable, as they allow exploration of interaction patterns when specific components (e.g. prey evolution e.g. via bottle-necks) change. More important, we were particularly interested in using ancestral populations in our work since "ancestral" refers to the fact that the individuals in neither population had a previous experience on confronting each other. This situation, that individuals never interacted before, is seldom observed in natural communities, which is an additional important advantage of our study. The microbial system we studied allowed us to explore how predator-prey communities co-evolve from a starting point where only ecological factors rule the interaction, but soon evolution starts to affect the interaction dynamics (Hiltunen et al., 2018) resulting in important changes in the observed dynamics. Thus, ancestral populations were considered as a starting point for the modelling work and these individuals were used for identifying the ecological model representing an unevolved predator-prey interaction.

In our theoretical approach, we compare the model results to the observational data to investigate which model has the highest explanatory power. To cover all relevant scenarios, we build four different versions of our model. We begin with an ecological model in which bacterial prey and the ciliated predator only interact. Relevant parameters like growth rate, initial defence level, or predation rate were estimated from the original data (Hiltunen et al., 2018) and additional experiments. After reinvestigation of the results, we stepwise add evolution to the model in form of only prey evolution, only predator evolution and co-evolution. Including the one-sided evolutionary models (prey and predator evolution only, respectively) helps us in evaluating the relative importance of prey and predator in the final co-evolutionary model. While the model with prey evolution explains most of the observed dynamics, the co-evolution model gives the best fit with the data, indicating predator evolution to be an additional factor.

\section{Material and methods}

\subsection{Microcosms experiments and data}

The parametrization of the model is based on controlled microcosm experiments with initially unexperienced (ancestral) individuals both in predator and prey species (see Supplementary material Data in Kaitala et al.xlsx). As a prey species the bacterial strain $P$. fluorescens strain SBW25 was used and the ciliated protozoan $T$. thermophila as a predator. All three treatments were started from one clonal isolate of bacteria to minimize initial genetic variability in populations. Experiments lasted 66 days. The experimental data were collected from microcosm experiments with a sequence of 48 -h batch processes. At the end of each 48-h growth period, $1 \%$ of each culture was transferred to a new vial containing fresh culture medium (King's B medium at concentration of 5\%) to initiate a new 48-h growth period (see Predator prey replicates 1-3 in Supplementary material Data in Kaitala et al.xlsx). The carrying capacity was estimated using data from three experiments without the predator (see Bacteria only in Supplementary material Data in Kaitala et al.xlsx).To estimate growth rate we isolated 5 ancestral and 5 evolved bacteria clones. The increase was measured every 5 min from growing population over $25 \mathrm{~h}$ (see Bacteria only in Supplementary material Data in Kaitala et al.xlsx). The obtained exponential growth curves were used to estimate the maximum growth rate.

\subsection{Ecological dynamics of the prey-predator system}

We begin by building a modified Lotka-Volterra predator prey model (Abrams, 2000) where a bacterial population is harvested by a ciliate population. Due to the observed limits in the bacterial population size in the data, we have replaced linear bacterial growth by density dependent logistic growth with a well-defined carrying capacity. Due to the high mortality rate caused by the sampling techniques ( $1 \%$ transfer every $48 \mathrm{~h}$ ), the natural mortality of the ciliate is omitted. We assume Type 1 functional response which is linear with respect the bacterial population size, based on additional data indicating that bacterial populations under these conditions (5\% King's B) never reach densities where ciliates are unable to consume more. Thus, we consider the following continuous-time model for the prey-predator interaction

$$
\begin{aligned}
& \frac{d P(t)}{d t}=r_{P}\left(1-\frac{P(t)}{K}\right) P(t)-a_{0} P(t) Z(t) \\
& \frac{d Z(t)}{d t}=b_{0} a_{0} P(t) Z(t),
\end{aligned}
$$

where $P(\mathrm{t})$ and $Z(\mathrm{t})$ are the densities of the prey and predator at time $t$, respectively. In the prey growth dynamics (Eq. (1)) $r_{P}$ is the density independent per capita growth rate and $K$ is the carrying capacity. The bacterial prey is attacked by the ciliate predator (Eq. (2)). Parameter $a_{0}$ denotes the attack rate, and $b_{0}$ is the conversion rate of prey to predator growth. In the ecological model, the life history traits and other ecological parameters are fixed in time.

\subsection{Co-evolutionary dynamics of the prey-predator system}

In formulating the model of co-evolutionary dynamics we utilize the well-known quantitative genetics approach Abrams et al., 1993; Mougi, 2010; Mougi and Iwasa, 2011). In this model class, each species has a trait which controls the life history parameters and the interaction between the species. These traits are subject to the evolution/adaptation in the course of the antagonistic interaction. The evolution is driven in each population by maximizing the fitness of the particular population. It should be noted that the traits of the species may control different parameters but that they can also control a specific parameter jointly. In the predator-prey model (Eqs. (1) and (2)) the potentially evolving parameters are bacterial growth rate, carrying capacity, attack rate and conversion rate. The data on the growth rates indicate that there is no difference between the maximum growth rates of ancestral prey and experienced prey. After considering the carrying capacity we noticed that the contribution of the carrying capacity to the evolution of the prey-predator dynamics is negligible. We admit that the evolution of both of these parameters may be a possible option but we assume here that these parameters do not contribute to the evolution of the prey-predator system in this particular case. We next present the model version which explain the co-evolutionary process best.

Let $u$ and $v$ denote the traits of the prey and predator, respectively. These traits are comparable to anti-predation investment (e.g. defence level) and investment into attack rate (e.g. handling time). The attack rate $a$ is assumed to be a function of the trait $u$ of the prey and the trait $v$ of the predator. An increase in the prey 
Table 1

Goodness of fit and estimated parameter values in the different models.

\begin{tabular}{lllllll}
\hline Evolution & Error E & $\mathrm{g}$ & $\mathrm{c}_{1}$ & $\mathrm{c}_{2}$ & $\mathrm{G}_{\mathrm{P}}$ & $\mathrm{G}_{\mathrm{Z}}$ \\
\hline No evolution & 3.8155 & & & & 0 & 0 \\
Only prey evolves & 0.9880 & 6.8666 & 1.1059 & 0.4026 & 0.0014 & 0 \\
Only predator evolves & 3.1196 & 6.2338 & 0.7980 & 0.5995 & 0 & 0.0432 \\
Both evolve & 0.8076 & \multirow{2}{*}{7.3347} & 0.8568 & 0.4745 & 0.0017 & 0.0271 \\
\hline
\end{tabular}

trait $u$ can be considered as an investment against the predator. As $u$ increases the attack rate decreases and the defence against predation is improved. However, the predator can also invest into the attack rate: an increase in the predator trait $v$ increases the attack rate and the defence against predation weakens. Thus, we assume that

$a(u, v)=a_{0} \exp (-g u) \exp \left(c_{1} v\right)$

where $a_{0}$ is the initial attack rate corresponding to the interaction between ancestral populations and $g$ and $c_{1}$ are fixed parameters.

Similarly, we assume that the trait $v$ of the predator affects the conversion rate:

$b(v)=b_{0} \exp \left(-c_{2} v\right)$

where $b_{0}$ is the initial conversion rate of an ancestral predator and $c_{2}$ is a parameter. Thus, the predator faces the trade-off between more efficient resource use in reproduction and the attack efficiency. This trade-off can be considered as energy allocation. Energy from food resource can either be allocated into agility which would increase the attack efficiency or into reproduction resulting in higher off-spring production.

The population dynamics of the prey and predator are given as

$$
\begin{aligned}
& \frac{d P(t)}{d t}=r_{P}\left(1-\frac{P(t)}{K}\right) P(t)-a(u, v) P(t) Z(t)=W_{P}(u, v) P(t) \\
& \frac{d Z(t)}{d t}=b(v) a(u, v) P(t) Z(t)=W_{Z}(u, v) Z(t),
\end{aligned}
$$

The trait dynamics includes the traits equations as follows (Abrams et al., 1993; Mougi, 2010).

$$
\begin{aligned}
\frac{d u(t)}{d t} & =G_{P} \frac{d W_{P}(u, v, t)}{d u} \\
& =G_{P}\left[a_{0} \exp \left(c_{1} v(t)\right) \exp (-g u(t)) Z(t)\right], u(0)=0, \\
\frac{d v(t)}{d t}= & G_{Z} \frac{d W_{Z}(u, v, t)}{d v} \\
= & G_{Z}\left[\left(c_{1}-c_{2}\right) b_{0} \exp \left(-c_{2} v(t)\right) a_{0} \exp \left(c_{1} v(t)\right) \exp (-g u(t)) P(t)\right], \\
& v(0)=0,
\end{aligned}
$$

where $G_{P}$ and $G_{Z}$ are parameters determining the speed of the evolution of the traits. The initial values of the traits $u$ and $v$ are chosen to be equal to 0 due to the fact that the ancestral individuals in each species have no earlier history of occurring together in a predator-prey interaction.

\subsection{Parameter estimation}

Fitting the model to the data requires the estimation of the constant parameters $r_{P}, K, \mathrm{a}_{0}, \mathrm{~b}_{0}, g, c_{1}, c_{2}, G_{P}$ and $G_{Z}$. The estimated parameters are given in Table 1.

To estimate maximum growth rate $r_{P}$, we fitted the exponential growth model $P(t)=P(0) \exp \left(r_{p} t\right)$ to the growth data (Bacteria only in Supplementary material Data in Kaitala et al.xlsx). We obtained an estimate $r_{P}=3.3 /$ day.

The carrying capacity was estimated from the observational data of $P$. fluorescens populations in the absence of the predator (Bacteria only in Supplementary material Data in Kaitala et al.xlsx). In the absence of predation, the population level of the P. fluorescens stabilizes to the carrying capacity $K=2.58 \times 10^{8}$ cells $/ \mathrm{ml}$ (see also Fig. 1a in the reference study (Hiltunen et al., 2018)).
The estimates for the parameter values of $a_{0}$ and $b_{0}$ were obtained by using the data of the second sampling period of the observational data (Predator prey replicates 1-3 in Supplementary material Data in Kaitala et al.xlsx). The values of the first sample at time $48 \mathrm{~h}$ were divided by 100 to obtain the initial values of the second growth period. The parameter values of $a_{0}$ and $b_{0}$ were estimated by simulating the ecological differential equation model (1) and (2) over one growth period of $48 \mathrm{~h}$ and by applying Monte Carlo method. The best estimate minimized the relative error at the end of the second sampling period

$e=\sqrt{\left((\Delta P)^{2}+(\Delta Z)^{2}\right)}$

where the normalized error value is $\Delta P=\left(\left(P_{o b s}-P_{\text {sim }}\right) / \max \left(P_{o b s}\right)\right.$ and $\Delta Z$ likewise, where "obs" and "sim" refer to observed and simulated values. We get $a_{0}=4.20 \times 10^{-6}$ and $b_{0}=5.75 \times 10^{-4}$. These parameter values were used in the ecological model as well as the initial values for the evolutionary models.

The rest of the parameters, $g, c_{1}, c_{2}, G_{p}$ and $G_{Z}$, for all the three evolutionary models were estimated applying Monte Carlo parameter estimation method using all the observational data. In each run, the parameters were sampled from an even distribution $[0.5 \times \mathrm{p}, 1.5 \times \mathrm{p}]$, where $\mathrm{p}$ is a fixed initial guess for the parameter. The error measure used is given as

$E=\sqrt{\sum\left((\Delta P(i))^{2}+(\Delta Z(i))^{2}\right)}$

where normalized error values are $\Delta P(i)=$ $\left(P_{o b s}(i)-P_{\text {sim }}(i)\right) / \max \left(P_{o b s}\right)$ and $\Delta Z(i)$ likewise. The parameters estimations and the error measure are given in Table 1 , where the error measure of the ecological model is also shown.

We validated our approach by fitting an additional model allowing the evolution in the carrying capacity in a similar approach as described above. This did not indicate any significant improvement in model explanation, so we decided to exclude the evolution in the carrying capacity from our model.

\section{Results}

All our models initially simulate the first few observations reasonably well. However, we see deviations from the observational data at later time points (Fig. 1). The ecological model without evolution fails to reflect the observed dynamics (Fig. 1a) and the model fit with the data is comparably poor (error $E$ minimized, $E==3.8155$ ). The ecological model fails in predicting the transients in the population densities. Particularly, the prey densities are predicted to be much lower than the observed densities. The equilibrium density of the bacterial population in the observational data is at $P=2 \times 10^{8}$ cells $/ \mathrm{ml}$, while the model predicts densities below $P=1 \times 10^{8}$ cells $/ \mathrm{ml}$. The predator density is predicted by the model to be at $Z=3 \times 10^{4}$ cells $/ \mathrm{ml}$ while in the observational data the predator density is much lower $\left(Z=1.1 \times 10^{4}\right.$ cells $\left./ \mathrm{ml}\right)$.

The model with prey evolution simulates the observational data better than without evolution (Fig. 1b) resulting in an improvement of the model fit $(E=0.9880)$. During the mid-period of the experiment, the model overestimates the predator densities. The final prey density is simulated higher than expected. Prey evolution 

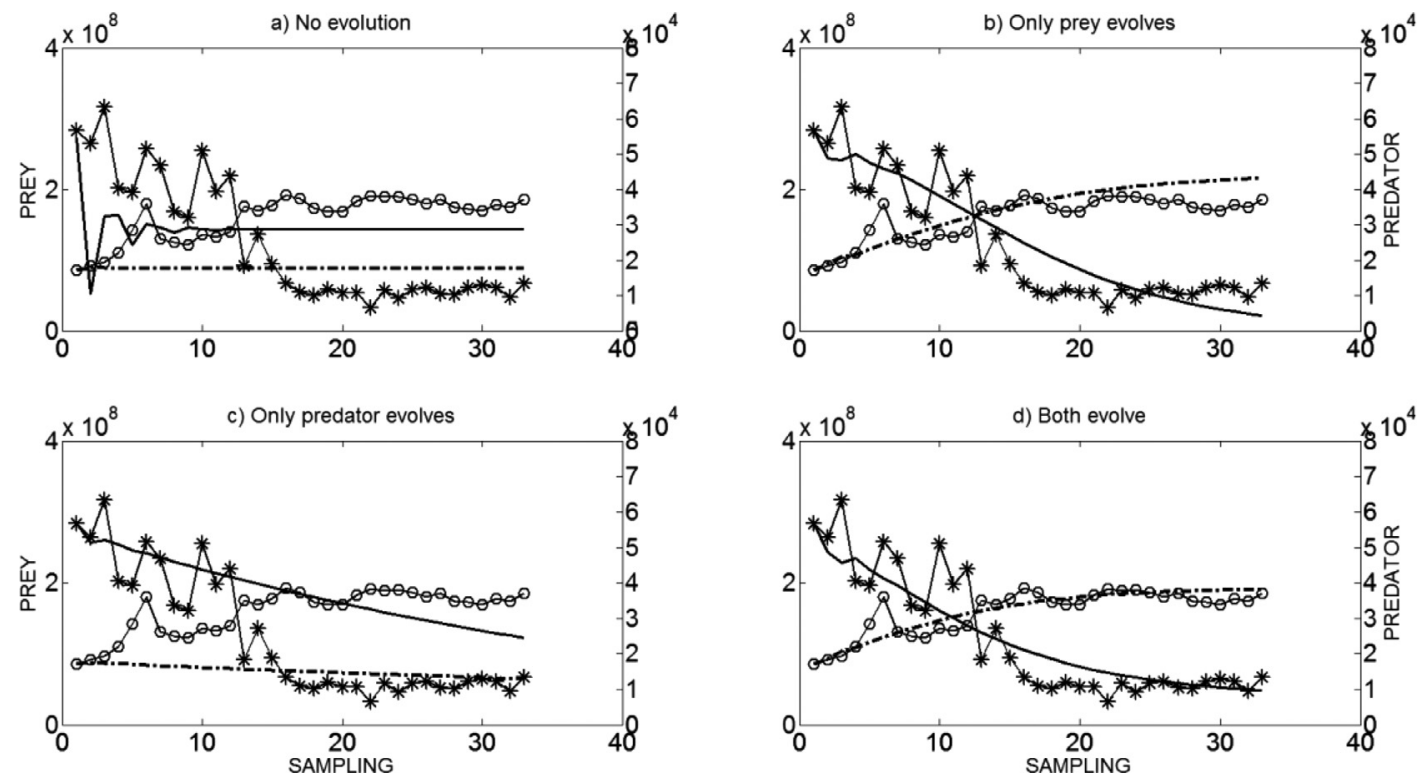

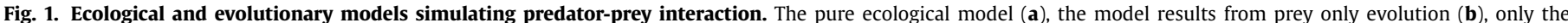



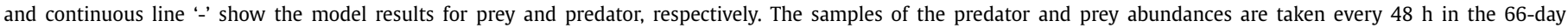

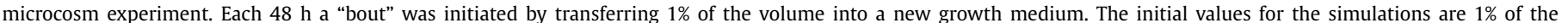
first observed values in the experiments.

a)
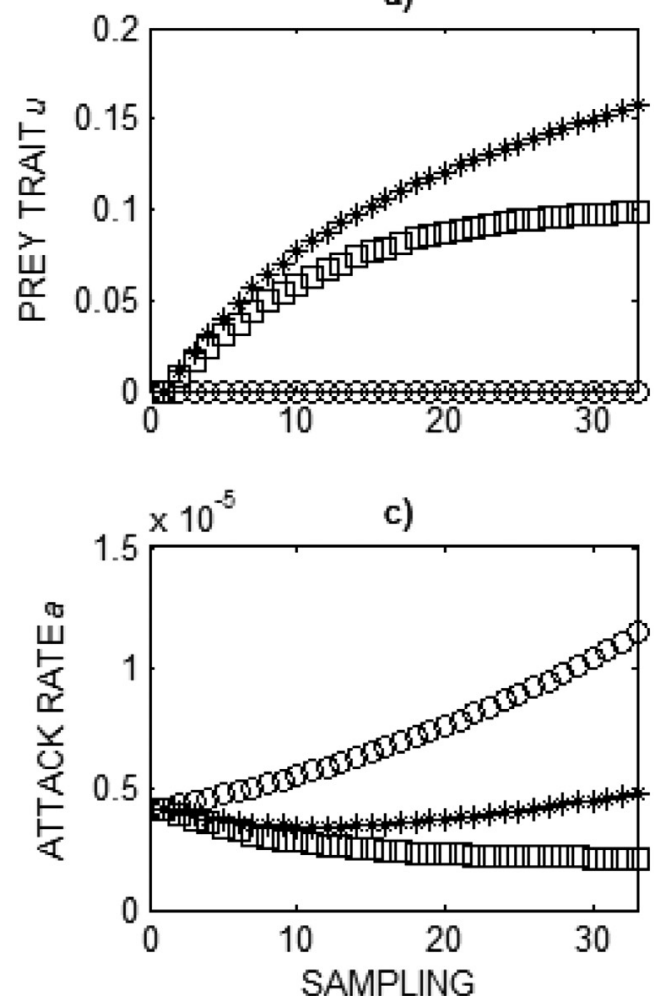

b)
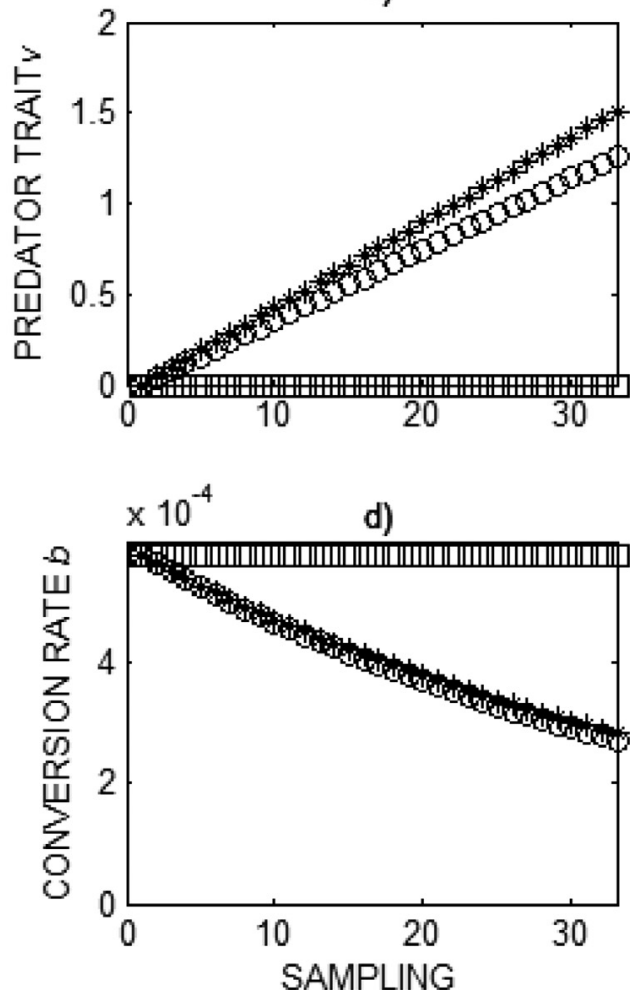

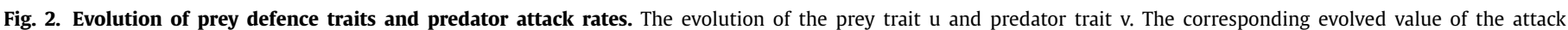
rate $a$ and conversion rate $b$. Squares $(\square)$, prey evolution model; circles (o), predator evolution model; stars $\left({ }^{*}\right)$, co-evolution model.

slows down after an initial increase, which might be responsible for this pattern (Fig. 2).

The model with predator evolution does not reflect the dynamics either (Fig. 1c) and we see a poor model fit again $(E=3.1196)$. Under this scenario the model predicts prey density to be far below the observed densities. The simulated predator density follows the observational densities reasonably well. The attack trait and the conversion trait change with opposing trends (Fig. 2).

Compared to the previous models, where no evolution occurred or only one partner evolved, the co-evolution model best simulates the observations (Fig. 1d) and we achieve the best model fit $(E=0.8076)$. The results represent a transition from the initial 
condition towards seemingly stabilized pairwise interaction where both species survive. Notably, the fit between the observational data and the model is very good. Both predator and prey traits increase monotonically without any sign of stabilization or lowering down the rate of increase (Fig $2 \mathrm{a}$ and $\mathrm{b}$ ). The attack rate first decreases but begins to increase again towards the end (Fig. 2c). The conversion rate decreases monotonically from the beginning (Fig. 2d). The error $E$ minimized between the observations and simulations of the co-evolution model is the smallest of all models and we suggest that this model is the best model to explain the observations. Our study indicates that prey evolution is a very important process in this system (prey evolution model has an error $E=0.9875$ compared to the model without evolution with $E=3.8155)$. However, both the smallest error $E$ and the change in prey and predator traits are strong indication that co-evolution of the predator is an additional important factor.

\section{Discussion}

Co-evolution between the two species can be detected using a most simple class of predator-prey models and appropriate model setting. We are able to explain the transient dynamics in the predator-prey interaction of the observational data using the tradition of quantitative co-evolutionary models (Mougi, 2010; Mougi and Iwasa, 2011). Our model predicts the observations of the original study (Hiltunen et al., 2018) accurately and highlights how the parameters change over time for both species, which indicates co-evolution. Two major conclusions can be drawn from these results. First, evolution of the prey has great impact on the trophic interaction, which is in line with the original study (Hiltunen et al., 2018). The prey benefits from the evolution as its density is multiplied compared to the non-evolutionary situation. Second, our approach suggests that co-evolution in the predator is an additional important detail in this system which is in line with other findings (Hiltunen and Becks, 2014).

Overall, our approach suggests that co-evolution can play a crucial role even in simple ecological systems, as we need both, ecological and evolutionary theory, to explain the observations. This is in line with previous findings, which showed that evolution of the prey explained the phase shift in rotifer-algae communities (Fussmann et al., 2003; Yoshida et al., 2003). However, here evolution of only one partner, the prey, was enough to explain the observations. In this system genetically diverse prey quickly evolved with one subpopulation being little defended but fast growing and a slow growing but well defended subpopulation, respectively. This difference compared to our study might be explained by the different experimental setting. The authors of the rotifer-algae study used a chemostat system which maintained the populations at constant dilution rates. This might result in maintaining prey diversity because both algae subpopulations experienced intraspecific competition. The rotifers might not need to evolve, as edible prey was, at least periodically, available throughout the experiment. In the experiment we simulated, populations were transferred to fresh condition at high rates with low inoculum volume (1\%). We speculate that this might end up with prey experiencing little intraspecific competition due to the repeated low densities, experiencing mainly directional selection.

We have assumed that the traits of the predator and prey affect the attack rate, the conversion rate and the defense level. A trade-off was only assumed for the predator such that increasing the attack rate decreases the consumption rate. It is also possible that the carrying capacity or the growth rate of the prey would be affected by a trade-off or even evolve. We added trade-offs and evolution in these traits in additional models, however, these additional variables did not provide any substantial improvement, thus we decided to exclude them from our model.
We assumed Type 1 functional response to model the harvesting of the prey by the predator. Type 1 functional response worked sufficiently well for the population level produced in the experiments. A linear response of ancestral $P$. fluorescens bacteria consumed by the $T$. thermophila ciliates at low bacterial densities has been verified experimentally by other works (Hiltunen et al., 2018). When the environment changes, for example with increasing concentration of nutrients, the population densities may increase, and the functional response may saturate. Consequently, the shape of the functional response may change (Gentleman et al., 2003). Such changes may lead to complex but sustained coexistence of the prey and predator (Mougi, 2010; Scheuerl and Stelzer, 2019).

From the modeling point of view it is obvious that when number of parameters increase (as occurs when we move from the ecological model to evolutionary model) the chances to improve the fit of the model to the data increase. Thus, one might argue that increasing the complicatedness of the model does the thing, producing nothing new. Ultimately, the challenge remains, however, to identify the processes that are crucial and behind the data. We argue here that considering the predator-prey interaction as an coevolutionary process and allowing the change of ecological parameters be explained as well defined evolutionary processes is a qualitatively different method than increasing parameters to the original ecological model.

Our approach suggests that ecology and evolution both interact in shaping community dynamics (Pelletier et al., 2009). Ignoring the importance of evolution might lead to false conclusions (Ellner et al., 2011). Particularly in experimental systems, species densities may fluctuate due to experimental conditions (e.g. lag periods and transfers) and deviation from expectations might be interpreted not as evolution. However, the observational data we simulated used microorganisms which are known to evolve quickly, which supports our conclusion.

\section{Funding}

This work was supported by an Academy of Finland (grant number \#1267541 to V.K.).

\section{Declaration of Competing Interest}

The authors declare no conflict of interest.

\section{Supplementary materials}

Supplementary material associated with this article can be found, in the online version, at doi:10.1016/j.jtbi.2019.110095.

\section{CRediT authorship contribution statement}

Veijo Kaitala: Supervision, Conceptualization, Methodology, Investigation, Software, Writing - original draft, Writing - review \& editing. Teppo Hiltunen: Conceptualization, Resources, Investigation, Writing - original draft. Lutz Becks: Conceptualization, Resources, Investigation, Writing - original draft. Thomas Scheuerl: Conceptualization, Methodology, Software, Investigation, Resources, Writing - original draft, Writing - review \& editing.

\section{References}

Abrams, P.A., 2000. The evolution of predator-prey interactions: theory and evidence. Annu. Rev. Ecol. Syst. 31, 79-105. https://doi.org/10.1146/annurev.ecolsys. 31.1.79.

Abrams, P.A., Matsuda, H., Harada, Y., 1993. Evolutionarily unstable fitness maxima and stable fitness minima of continuous traits. Evol. Ecol. 7, 465-487. https: //doi.org/10.1007/BF01237642.

Buckling, A., Rainey, P.B., 2002. Antagonistic coevolution between a bacterium and a bacteriophage. Proc. Biol. Sci. 269, 931-936. https://doi.org/10.1098/rspb.2001. 1945. 
Ellner, S.P., Geber, M.A., Hairston Jr, N.G., 2011. Does rapid evolution matter? Measuring the rate of contemporary evolution and its impacts on ecological dynamics. Ecol. Lett. 14, 603-614. https://doi.org/10.1111/j.1461-0248.2011.01616.x.

Fiegna, F., Scheuerl, T., Moreno-Letelier, A., Bell, T., Barraclough, T.G., 2015. Saturating effects of species diversity on life-history evolution in bacteria. Proc. R. Soc. B 282, 20151794. https://doi.org/10.1098/rspb.2015.1794.

Friman, V.-P., Buckling, A., 2013. Effects of predation on real-time host-parasite coevolutionary dynamics. Ecol. Lett. 16, 39-46. https://doi.org/10.1111/ele.12010.

Fussmann, G.F., Ellner, S.P., Hairston Jr, N.G., 2003. Evolution as a critical component of plankton dynamics. Proc. R. Soc. Lond. B 270, 1015-1022. https://doi.org/10. 1098/rspb.2003.2335.

Fussmann, G.F., Loreau, M., Abrams, P.A., 2007. Eco-evolutionary dynamics of communities and ecosystems. Funct. Ecol. 21, 465-477. https://doi.org/10.1111/j. 1365-2435.2007.01275.x.

Gause, G.F., 1934. Experimental analysis of Vito Volterra's mathematical theory of the struggle for existence. Science 79, 16-17. https://doi.org/10.1126/science.79. 2036.16-a.

Gentleman, W., Leising, A., Frost, B., Strom, S., Murray, J., 2003. Functional responses for zooplankton feeding on multiple resources: a review of assumptions and biological dynamics. In: Deep Sea Res. Part II Top. Stud. Oceanogr., The US JGOFS Synthesis and Modeling Project: Phase II, 50, pp. 2847-2875 https://doi.org/10. 1016/j.dsr2.2003.07.001.

Gómez, P., Buckling, A., 2011. Bacteria-phage antagonistic coevolution in soil. Science 332, 106-109. https://doi.org/10.1126/science.1198767.

Hairston Jr, N.G., Ellner, S.P., Geber, M.A., Yoshida, T., Fox, J.A., 2005. Rapid evolution and the convergence of ecological and evolutionary time. Ecol. Lett. 8, 11141127. https://doi.org/10.1111/j.1461-0248.2005.00812.x.

Hiltunen, T., Becks, L., 2014. Consumer co-evolution as an important component of the eco-evolutionary feedback. Nat. Commun. 5, 5226. https://doi.org/10.1038/ ncomms6226.

Hiltunen, T., Cairns, J., Frickel, J., Jalasvuori, M., Laakso, J., Kaitala, V., Künzel, S., Karakoc, E., Becks, L., 2018. Dual-stressor selection alters eco-evolutionary dynamics in experimental communities. Nat. Ecol. Evol. 12, 1974-1981. https: //doi.org/10.1038/s41559-018-0701-5.
May, R.M., 1972. Limit cycles in predator-prey communities. Science 177, 900-902. https://doi.org/10.1126/science.177.4052.900.

Mougi, A., 2010. Coevolution in a one predator-two prey system. PLoS ONE 5. https: //doi.org/10.1371/journal.pone.0013887.

Mougi, A., Iwasa, Y., 2011. Unique coevolutionary dynamics in a predator-prey system. J. Theor. Biol. 277, 83-89. https://doi.org/10.1016/j.jtbi.2011.02.015.

Pelletier, F., Garant, D., Hendry, A.P., 2009. Eco-evolutionary dynamics. Philos. Trans. R. Soc. B Biol. Sci. 364, 1483-1489. https://doi.org/10.1098/rstb.2009.0027.

Rosenzweig, M.L., 1971. Paradox of enrichment: destabilization of exploitation ecosystems in ecological time. Science 171, 385-387.

Scheuerl, Cairns Johannes, Lutz, Becks, Teppo, Hiltunen, 2019. Predator coevolution and prey trait variability determine species coexistence. Proc. R. Soc. B Biol. Sci. 286, 20190245. https://doi.org/10.1098/rspb.2019.0245.

Scheuerl, T., Stelzer, C.-P., 2019. Asexual reproduction changes predator population dynamics in a life predator-prey system. Popul. Ecol. 34, 210-216. https://doi. org/10.1002/1438-390X.1017.

Scheuerl, T., Stelzer, C.-P., 2013. Patterns and dynamics of rapid local adaptation and sex in varying habitat types in rotifers. Ecol. Evol. 3, 4253-4264. https://doi.org/ 10.1002/ece3.781.

Schoener, T.W., 2011. The newest synthesis: understanding the interplay of evolutionary and ecological dynamics. Science 331, 426-429. https://doi.org/10.1126/ science.1193954.

Volterra, V., 1926. Fluctuations in the abundance of a species considered mathematically [WWW Document]. Nature. https://doi.org/10.1038/118558a0.

Wiser, M.J., Ribeck, N., Lenski, R.E., 2013. Long-term dynamics of adaptation in asexual populations. Science 342, 1364-1367. https://doi.org/10.1126/science. 1243357.

Yoshida, T., Jones, L.E., Ellner, S.P., Fussmann, G.F., Hairston Jr, N.G., 2003. Rapid evolution drives ecological dynamics in a predator-prey system. Nature 424, $303-$ 306. https://doi.org/10.1038/nature01767. 